\title{
Morphogenesis of 'decapitated' spermatozoa in a man
}

\author{
B. Baccetti, M. G. Selmi and P. Soldani \\ Institute of Zoology, University of Siena, 53100 Siena, Italy
}

\begin{abstract}
Summary. Study of biopsy specimens of a man with 'decapitated' spermatozoa indicated that the malformation was due to an overproduction of a membranous vesicles system by the Golgi complex in the region between the centrioles and acrosome during the spermatid stage. The tails were almost normal in structure and $50 \%$ were motile in the ejaculate although few followed a straight trajectory.
\end{abstract}

\section{Introduction}

The 'decapitated sperm' defect has been reported for bulls (Hancock \& Rollinson, 1949; Blom \& Birch-Andersen, 1970) and man (Perotti, Giarola \& Gioria, 1981). The ejaculate contains motile tails detached from the heads, which are irregularly shaped, with various degrees of maturity in the chromatin. The tails contain normal axonemes, while mitochondria are frequently displaced in an anterior knob. The origin of the defect is still unknown, although Blom \& Birch-Andersen (1970) suggest that the detachment in bulls occurs in the epididymis. We have now studied biopsy material obtained from the testis and the nasal ciliated epithelium of an infertile man affected by the 'decapitated sperm' defect.

\section{Materials and Methods}

The patient was a 35-year-old man referred for infertility. Three ejaculates, obtained by masturbation, were studied by various techniques.

Scanning electron microscopy. The cells, isolated by centrifugation for $10 \mathrm{~min}$ at $1000 \mathrm{~g}$, were fixed in $1 \%$ glutaraldehyde for $1 \mathrm{~h}$, centrifuged, washed in distilled water, placed on aluminium disks and dried by the critical-point method. The disks were mounted on the mounting base, shadowed with carbon and with an $\mathrm{Au} / \mathrm{Pt}$ alloy and then examined with an SEM 2 Jeol.

Transmission electron microscopy. The cells of the ejaculates were fixed in Karnovsky's fixative for $1 \mathrm{~h}$ and then centrifuged for $10 \mathrm{~min}$ at $1000 \mathrm{~g}$, washed in cacodylate buffer, post-fixed for $1 \mathrm{~h}$ in $1 \% \mathrm{OsO}_{4}$, dehydrated and embedded in Epon-Araldite. Thin sections were cut with an LKB microtome and stained with uranyl acetate and lead citrate before observation in a Philips 301 electron microscope.

Cinematographic recording. Ejaculates of a normal 37-year-old man and of the patient were diluted with $0.9 \%(\mathrm{w} / \mathrm{v}) \mathrm{NaCl}$ buffered at $\mathrm{pH} 7.5$ with $0.005 \mathrm{M}$-Soerensen's phosphate buffer, a solution which has given us good results in previous studies of mammalian sperm motility (Baccetti, Renieri, Rosati, Selmi \& Casanova, 1977). Then the sperm suspension was placed on a slide and examined under a coverslip with dark-field cinematographic recordings. Recordings at 18 frames/ sec were obtained with a negative Kodak film ( 400 ASA sensitivity) and a PL $25 \times$ N.A. 0.50 Leitz objective. Films of 25 normal human spermatozoa and of 25 selected spermatozoa of our patient 
were then projected for analysis of movement. Sperm rotation was evaluated by identification of profile position; sperm trajectory and swimming speed were studied by following the displacement of the anterior end of the cell.

Biopsy material. Fragments of tissue obtained by biopsy from the testis and the nasal ciliated epithelium were examined by transmission electron microscopy, as above, after fixation in $2.5 \%$ glutaraldehyde for $2 \mathrm{~h}$ and post-fixation in $1 \% \mathrm{OsO}_{4}$ for $1 \mathrm{~h}$. Biopsy material from the nasal ciliated epithelium was also prepared for scanning electron microscopy as above.

\section{Observations}

Ejaculate. The observations made by light and electron microscopy largely confirmed those of Perotti et al. (1981): elongated undulating tails and globular elements, considered to be sperm heads, were present. The sperm heads were present in numbers similar to those of the tails, i.e. about $14 \times 10^{6} / \mathrm{ml}$.

Transmission electron microscopy showed that the sperm heads had characteristics already described and that the tails (Pl. 1, Figs 1 \& 2) were of the three types (A, B, C), and in the same proportions, as described by Perotti et al. (1981). Half of the type B tails had an axoneme devoid of mitochondria and showed an evident spindle-shaped body (P1. 1, Fig. 1); the rest had a small number of scattered mitochondria concentrated in the apical knob (Pl. 1, Fig. 2), and had no spindle-shaped body.

About $50 \%$ of the tails were motile, and about $20 \%$ of these showed a linear trajectory.

All the isolated tails, whether moving linearly or irregularly, turned on the longitudinal axis. This fact was confirmed by the position analysis of the apical knob since it is located first on one side and then on the other side of the cell. A complete rotation of $180^{\circ}$ along the sperm axis was performed each $0.5 \mathrm{sec}$ and there were 6 waves $/ \mathrm{sec}$ from the anterior end. The effective velocity of the tails was about $20 \mu \mathrm{m} / \mathrm{sec}$.

\section{PLATE 1}

Fig. 1. Longitudinal section of a 'B' type tail. A cytoplasmic knob contains the initial portion of the tail. The connecting piece is rudimentary (CP). The middle piece is lacking. A spindleshaped body is associated with the tail (SB). The fibrous sheath (FS) is normal. $\times 20000$.

Fig. 2. Longitudinal section of a 'B' type tail, showing a few isolated mitochondria (M) present in the apical knob. The tail starts close to the proximal centriole (PC). A true middle piece is lacking. Only two mitochondria (M) flank the axoneme. $\times 27500$.

Fig, 3. Biopsy material. Early spermatid showing a bipartite Golgi complex (G) giving origin to an extensive system of vesicles in the region between the nucleus $(\mathrm{N})$ and centrioles $(\mathrm{C})$. $\times 20000$.

\section{PLATE 2}

Fig. 4. 'Decapitated sperm' defect. A spermatid from the biopsy of the same patient. Numerous vesicles arising from the Golgi complex $(\mathrm{G})$ are located between the nucleus $(\mathrm{N})$ and the developing tail $(\mathrm{T})$. The centriolar adjunct $(\mathrm{CA})$ associated with the proximal centriole (PC), the chromatoid body ( $\mathrm{Ch})$ and the annulus (An) are evident. $\times 30000$.

Fig. 5. Head of late spermatid. The nuclear membrane is redundant and shows pores (arrow). No tail could be found, in spite of careful examination of serial sections of such cells. $\times 20000$.

Fig. 6. Light micrograph of testicular material obtained from the biopsy in a patient having the 'decapitated sperm' defect. Spermatogonia $(\mathrm{Sg})$ and spermatocytes $(\mathrm{Sc})$ are normal. The late spermatids $(\mathrm{St})$ have a casual orientation. $\times 550$.

Fig. 7. Head of the late spermatid. The chromatin is normally condensed, even if several vacuoles (V) are present in the nucleus. The acrosome (A) is longer than normal and detached from the nucleus. The nuclear envelope seems to be incomplete or absent. Clusters of mitochondria $(\mathrm{M})$ are present in the perinuclear cytoplasm. $\times 25000$. 
PLATE 1
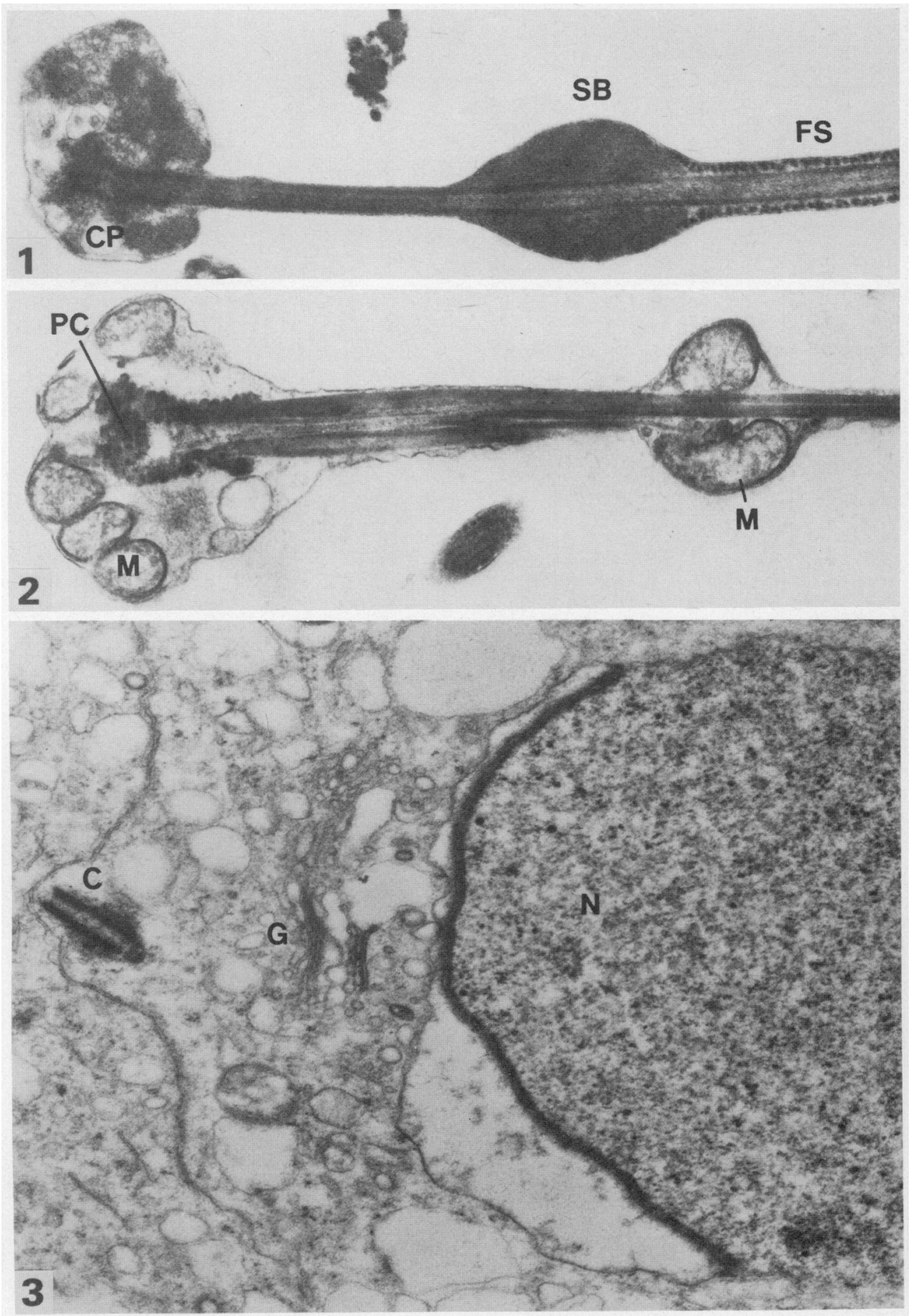


\section{PLATE 2}
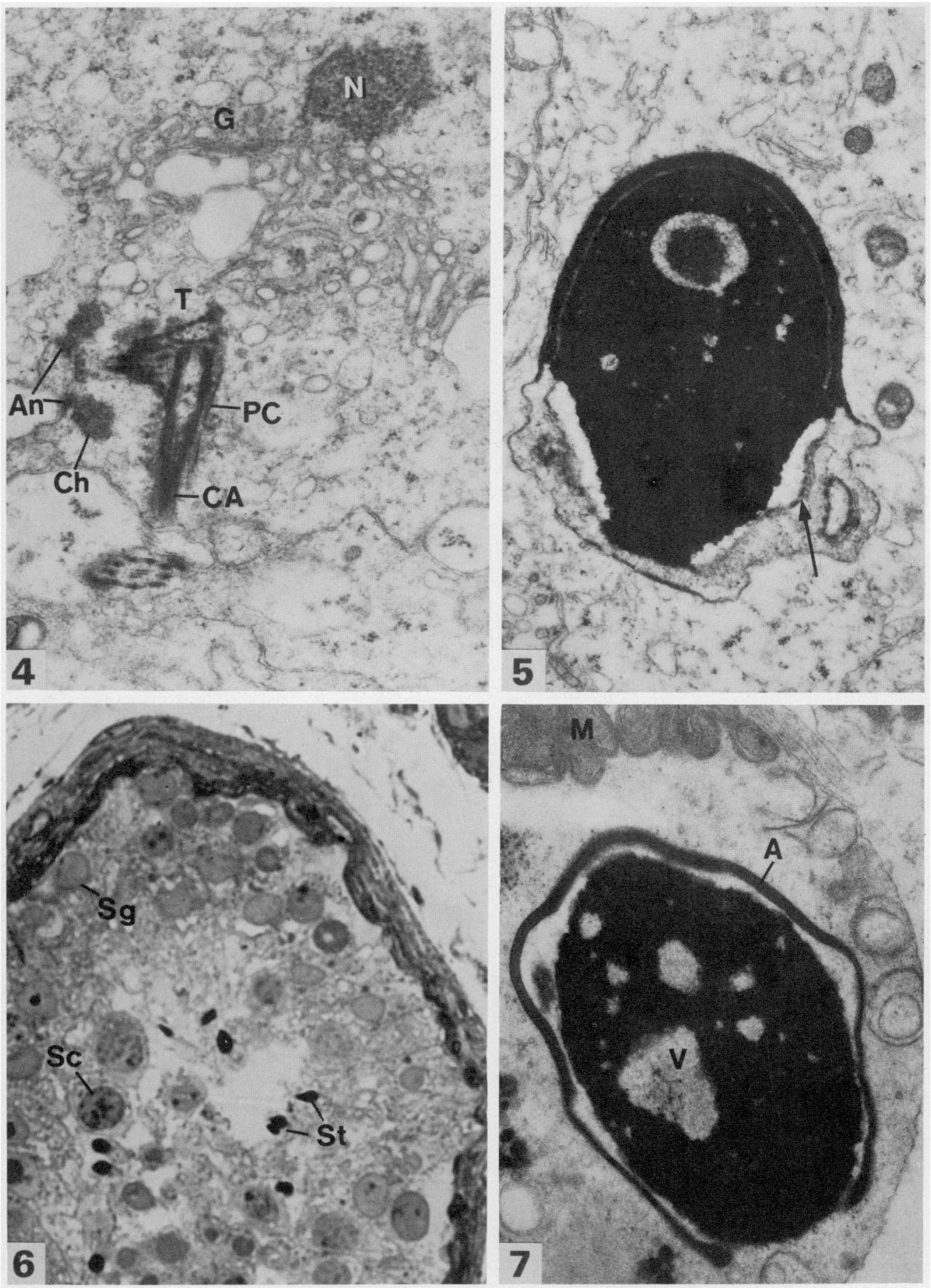
The equivalent values for a normal man were $>70 \%$ of motile spermatozoa, $\sim 80 \%$ of the motile spermatozoa had a linear trajectory, longitudinal rotation with a frequency of $6 / \mathrm{sec}$, wave propagation $(6 / \mathrm{sec})$ from the middle piece towards the end of the flagellum and an effective velocity of $\sim 50 \mu \mathrm{m} / \mathrm{sec}$.

Biopsy material. All the stages of spermiogenesis were present and apparently normal until the spermatid stage. The early spermatid was rounded with a nucleus full of granular chromatin, surrounded by mitochondria and a chromatoid body. Centrioles were located far from the nucleus (Pl. 1, Fig. 3), in the direction of the acrosomal area, and were separated from the nucleus and the juxtaposed acrosomal vesicle by the Golgi complex (Pl. 1, Fig. 3), which seemed to have two maturing faces, one convex and one concave, separated by a vesicle. Subsequent stages of spermiogenesis (PI. 2, Fig. 4) showed the nucleus and acrosome becoming more and more dense and compact, surrounded by microtubules and scattered mitochondria, and a developing tail, with striated columns and other normal accessory structures in a separate cytoplasmic area, limited by numerous Golgi cisternae. The late spermatids appeared to be of two types. The nucleated variety (Pl. 2, Figs 5 \& 7) included an acrosome, manchette, mitochondria and a system of annulate lamellae close to the posterior pole of the nucleus where the basal lamina and implantation fossa were absent, the nuclear envelope was discontinuous, and the posterior redundant area, where annuli are usually conserved, was lacking. Anucleated late spermatids contained centrioles, an axoneme, sometimes variously arranged mitochondria, a spindle-shaped body and always the fibrous sheath. Binucleate or multinucleate spermatids, also devoid of tails, were often seen. The mature heads present in the seminiferous tubules were located in the centre of the lumen but their orientation was not constant (Pl. 2, Fig. 6).

Nasal ciliated epithelium. The ciliated cells of this epithelium, examined by scanning and transmission electron microscopy, appeared absolutely normal.

\section{Discussion}

In this man the malformation leading to 'decapitated'spermatozoa occurred at the spermatid stage, and was due to an overproduction of a membranous vesicle system by the Golgi complex in the region between the centrioles and acrosome. The complete separation of the axonemal domain from the nucleated domain can occur after the mitochondria have been arranged around the axoneme, or before, when all mitochondria are still around the nucleus and the spindle-shaped body surrounds the axoneme. This explains the different tail models described in the mature spermatozoon. The detached heads exhibit random orientation in the seminiferous tubules. This disorientation is probably due to the loss of contacts between nucleus and axoneme, although Sertoli cells are also believed to play a role in spermatid orientation. The tails show mainly irregular motility.

The sperm heads were showing signs of alteration even when in the testis: the nuclear envelope was always incomplete, being absent from the posterior pole of the nucleus, the acrosomes were sometimes irregularly shaped, and 2 or 3 nuclei were frequently grouped in the same cytoplasm. The defect seems to be limited to the sperm flagellum: cilia of the nasal epithelium appeared normal, confirming that the 'decapitated sperm' condition in this man did not arise through a failure of the centrioles to migrate to their appropriate position in the cell.

\section{References}

Baccetti, B., Renieri, T., Rosati, F., Selmi, M.G. \& Casanova, S. (1977) Further observations on the morphogenesis of the round headed human spermatozoa. Andrologia 9, 255-264.

Blom, E. \& Birch-Andersen, A. (1970) Ultrastructure of the 'decapitated sperm defect' in Guernsey bulls. $J$. Reprod. Fert. 23, 67-72.
Hancock, J.L. \& Rollinson, D.H.L. (1949) A seminal defect associated with sterility of Guernsey bulls. Vet. Rec. 61, 742-743.

Perotti, M.L., Giarola, A. \& Gioria, M. (1981) Ultrastructural study of the decapitated sperm defect in an infertile man. J. Reprod. Fert. 63, 543-549. 